\title{
COLONIZATION OF CANDIDA AFTER THE PLACEMENT OF A TONGUE PIERCING, A CASE REPORT
}

\author{
SZENDE LEDAN-MUNTEAN ${ }^{1}$, BIANCA TUDOR ${ }^{2}$ \\ ${ }^{1,2}$ University of Medicine, Pharmacy, Science and Technology of Târgu-Mureș
}

\begin{abstract}
Keywords: $\quad$ tongue Abstract: The practice of piercing the body's different areas is a commonly observed form of body piercing, candidiasis, modification. It implies puncturing the skin tissue resulted in an opening, in which specific jewellery candida albicans, fungal or an intradermal implant could be inserted. The tongue piercing is a highly popular form of this infection practice, but can be followed by multiple acute and chronic complications, such as bacterial and fungal infections. Our case describes the clinical situation of a 25 year old immunocompetent woman, who sought medical help after observing tissue abnormalities, following a newly inserted tongue piercing. We identified a correctly and professionally placed tongue jewellery associated with all clinical hallmarks of oral Candidiasis. Our aim was to microbiologically identify and treat the present yeast colonies.
\end{abstract}

\section{INTRODUCTION}

Body piercing is a form of body modification, which presumes in the practice of puncturing or cutting a part of the body resulted in an opening, in which specific jewellery or an intradermal implant could be inserted. The practice of these types of body modifications has an ancient history, being used to claim status, belonging and beauty in distinct aboriginal tribes. Their purposes were multiple, serving spiritual, financial and attribution- related impetuses. In modern days, piercings are surrounded with great success. They became fashionable during the 1990's and kept recurring since, in different forms and inserted in unusual body parts. The tongue piercing is a highly popular form of this practice, surviving the always changing fashion waves.(1) It is the piercing that requires the most precision and knowledge regarding the placement technique, due to the highly vascularised constitution of the tongue, several technical errors are being registered throughout its popular years. The insertion of the jewellery in the tongue can be followed by multiple acute and chronic complications and literature describes numerous pathological issues following its practice.

These clinical manifestations can have great local and general health effects. The clinical picture may vary from local, systemic to combined implications. Immediate local responses presume in mild bleeding, inflammation, infection, acute pain and speech impairment. Long term local complications, such as cracks and fissures on the dental enamel, soft tissue destruction, nerve damage, loss of taste, chronic pain and bacterial, also fungal infections and can be observed in high incidences.(2-8)

Candida is the abbreviation used to describe a class of fungi that includes more than 150 species of yeast. In healthy individuals, Candida exists harmlessly on mucosal surfaces, like the gastrointestinal tract, mouth, nose, ears, eyes and reproductive organs. This type of fungi can also be observed on the skin. It is considered to serve a beneficial purpose in the body, although when the impairment of the normal flora occurs, it causes an overgrowth of a certain type of Candida, causing Candidiasis, or commonly, thrush. Oral candidiasis is a common infection of the buccal cavity caused by a colonization of Candida albicans. Its incidence is affected by age and predisposing factors. There are three groupings of this mycotic infection: acute or chronic candidiasis, and angular cheilitis. Risk factors inducing Candidiasis can be multiple; the impairment of salivary gland function, high carbohydrate diet, smoking, alcohol and drug use, dentures, certain immunosuppressing diseases and the local insertion of foreign objects, such as piercings can stimulate colonization. $(9,10)$

\section{AIM}

Our purpose was to microbiologically identify, differentiate and treat a candidiasis- like lesion, associated with a newly places tongue piercing.

\section{MATERIALS AND METHODS}

We present the case of a 25-year old, Caucasian, immunocompetent woman, who presented in our dental clinic, complaining about a thick white deposit on her tongue, associated with burning pain, altered sense of taste and difficulty swallowing. The patient described the appearance of this symptomatology following a tongue piercing insertion.

During the special examination of the oral cavity we observed a correctly inserted tongue piercing, surrounded by marked infection and a white- light yellow, plaque- like deposit, with extensive character, covering the surface of the ventral tongue. According to the patient, these symptoms appeared shortly, in 2 days, after the piercing insertion, although she followed the aftercare instructions given by the piercer professional: she avoided smoking and drinking alcohol for 48 hours, she consumed foods and drinks solely at room temperature, avoiding spicy and hard consistency aliments, she

${ }^{1}$ Corresponding author Ledan-Muntean Szende, P-ța Trandafirilor, Nr. 57/A, Tg.-Mureș, România, Email: dishyledan@gmail.com, Phone: +40799 646666

Article received on 24.07.2020 and accepted for publication on 02.12.2020 


\section{CLINICAL ASPECTS}

avoided oral contact with her partner and also rinsed her buccal cavity with alcohol free mouthwash, multiple times during a day.

The patient further described a burning sensation of the implicated area and the throat, also the impairment of food deglutition. The infection was localized, surrounding the newly inserted jewellery, marked by erythema, edema and pus secretion. We, as well, observed a circular, mild, lysis- like soft tissue regression on the tongue's ventral surface, which appeared to be caused by the upper ball shaped element of the jewellery, due to tissue constriction, resulted by the edema of the tongue.

Our clinic contacted the piercer, who confirmed the hygienic circumstances of the insertion, using pre-sterilized jewellery and utensils, also appraised us about the professional origin of the jewellery, which was manufactured of austenitic SAE 316 stainless steel, commonly known as surgical steel. This type of surgical steel serves biomedical application and also is frequently used in body jewellery fabrication, due to a high skin tolerance and strong biocompatibility.

The extensive examination of the oral cavity did not indicate any other sources that could cause the yeast colonization or the infection. Our patient presented a good general oral health, without any dental and soft tissue damages or salivary gland dysfunction.

The patient also presented mild flu- like symptoms, claiming that she felt generally ill the days prior, but denied administrating any kind of afferent medication.

Summing up the observed clinical manifestations and the associated generalized pathology, we suspected that our patient suffered from a locally manifested oral Candidiasis, induced by the placement and superinfection of the tongue jewellery, enhanced by the weakened immune- system, due to the mild upper respiratory issues she was presenting.

After the patient's informed consent, we performed a specimen collection of the suspected candida colonization on the lingual mucosa, using and oral swab and sent it for a microbiological examination, for determining the type of the colonized fungi.

The specimen was transported on Sabouraud media and cultivated below 37 degrees Celsius, followed by subcultivation of the newly grown fungi to cromogen agar CandiSelect plates for selective isolation of the specific yeasts, which allows to directly identify Candida albicans and presumptively differentiate Candida tropicalis, Candida glabrata și Candida krusei. All grown colonies were counted and identified by specific biochemical and growth marking characteristics.

We removed the implicated jewellery and referred our patient an adequate treatment plan. Fluconazole $100 \mathrm{mg}$. capsules were prescribed to be administrated orally, following a special scheme: on the first day of treatment administration of $300 \mathrm{mg}$. of substance, for initiating the dose, followed by 9 days, administrating $150 \mathrm{mg}$. of substance. Our treatment plan also targeted the localized, marked bacterial infection of the implicated area for which we prescribed a combined antibiotic treatment, consisting of Amoxicillin $875 \mathrm{mg}$. and Clavulanic acid $125 \mathrm{mg}$. This medication contains both excipients in one single $1000 \mathrm{mg}$ tablet and we suggested administrating 1 tablet per day, for a period of 10 days.

After 48 hours, we observed the growth of 4 colonies on the Sabouraud plates, which were characterized by whitishyellow creamy confluent plaques, resembling milk curds or cottage cheese. Subcultures on the specific agar plates presented blue- indigo colonies. After identifying on Vitek 2 system the specific biochemical and morfotinctorial characteristics of the growth, we concluded the certain presence of Candida albicans on the surface of our patient's tongue.

The symptomatology started regressing after 5 days under the specific pharmaceutical treatment, disappearing totally in 9 days.

Periodical examinations were taken into agenda. We observed our patient's evolution monthly and observed a full recovery of the pierced area in a total of 4 months, without the recurrence of the Candidiasis, during the observational period.

Figure no. 1. A- Frontal incidence of the affected ventral tongue, presenting a marked infection and clinical hallmarks of oral Candidiasis; B- 5 days under anti- fungal and antibiotic treatment, showing progressive regression of the infection; C- Patient's status after 4 months of ending treatment and removal of the jewelry

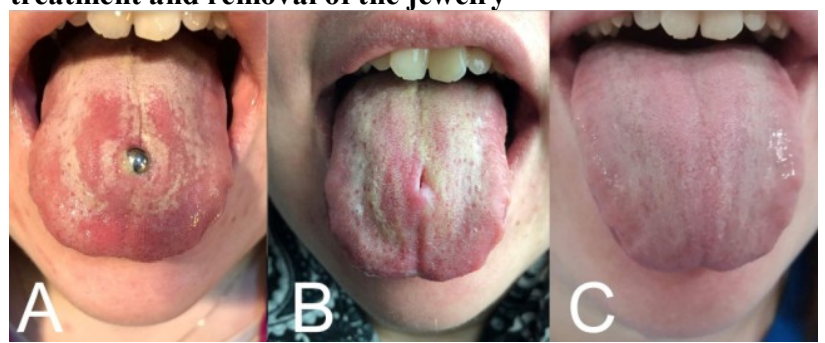

DISCUSSIONS

Oral Candidiasis has been known to occur in humans for over 2000 years, fact mentioned by Hippocrates, who described the pathology in his findings as a commonly present and superficial infection of the oral and vaginal mucosa. It was not until the mid-1800s, that documented research on characteristics and pathogenesis of Candida were initiated.(11) Bernhard von Langenbeck, a young university lecturer, is the first to publish in November 1839 about a fungus as aetiological agent of an oro-pharyngeal and oesophageal candidiasis.(12)

The most common yeast pathogen, Candida albicans, was identified in the 19th century. Barnett described in 2008, that in the early 1900s, C. albicans was observed in the buccal cavity of 2-6 weeks old and 1-6 year old children, the majority of them being rather healthy.(13) Later, studies revealed the normality of the oral carriage of C. albicans among healthy adults. Recently a marked escalation of the pathogenesis of this commensal has been observed due to changes in the practice of conventional medicine, such as introduction of larg -spectrum antibiotics, the higher incidence of risk factors, like smoking, alcohol and substance use, the prevalence of associated general health issues, such as Diabetes mellitus or AIDS and the practice of piercing the oral region.(14)

Conducted studies show, the Candida colonies were more prevalent among tongue-pierced than facial-pierced people, all deposits being identified as C. albicans.(15)

The bacterial infections associated to piercing insertion are common. The oral cavity is a complex and opportunistic environment for microbes. Many factors can influence the composition of the buccal microbial flora. Age, nutrition, oral hygiene, habitual vices, such as smoking and alcohol consumption, nevertheless the existing dental and periodontal diseases are considered determining factors.

Microorganisms that have a predilection for colonizing the tongue and oral mucosa include aerobic-, anaerobic streptococci and anaerobic Gram-positive bacilli. A newly inflected injury, such as jewellery insertion in the soft tissue, can represent a great opportunity for these microbes to colonize the affected area, strongly inhibiting the healing process. Total healing of the pierced area is described, by patients, to occur between 4-6 weeks, a period of time under which other associated pathology, such as Candida colonization 


\section{CLINICAL ASPECTS}

can develop.(16)

Post-operative indications, given by the piercer can reduce the incidence of developing associated pathology. Inchingolo et al. describe in their research paper some techniques that have been proved to ameliorate or even diminish the pathological consequences of oral piercings. In this paper we can read about a cold liquid diet followed by a soft food diet, applying ice externally for 30 minutes within every 45 minutes for 5 times a day. The paper describes the importance of mouth rinsing with $0,12 \%$ chlorhexidine solution for 5 times a day for the first ten days, also decreasing the consumption of alcohol, caffeine and reducing the number of smoked cigarettes per day. These substances are known to interfere with the epithelial reconstruction, hence decrease the possibility of healing. Replacement of the initial jewellery with a smaller one is, as well, indicated, alongside with aphonia as much as possible. Paying meticulous attention to oral hygiene, especially at the site of piercing is extremely important, as well is checking the status of the piercing as often as possible for the avoidance of infections.(17)

Although the occurring bacterial infections are treatable with common use broad- spectrum antibiotics, the administration of this type of medicine can significantly weaken the natural immune response of one's body, determining an opportunistic environment for several types of oral yeast colonization.

Diagnosis of oral thrush presumes in the identification of clinical symptoms, the visible presence of the candida colonies on the suspected mucosal surface, followed by of a specimen collecting smear or biopsy of the lesion, showing hyphae in the epithelium, positive culture, and specific serological tests. The importance of differentiating certain types of yeasts consists in the identification of NCAC species, such as C. glabrata, C. krusei and C. dubliniensis, which are naturally sensitive or resistant to some of the common antifungal drugs $(18,19)$. Oral C. albicans is known to respond well regressively to first line anti- fungal agents, such as Fluconazole, Clotrimazole troches, Nystatin suspension and Nystatin pastilles (20). In our patient's case Fluconazole was shown to diminish the Candidiasis symptomatology with success, even though the treatment was associated with a broad- spectrum antibiotic substance, for eradicating the associated bacterial infection.

\section{CONCLUSIONS}

In the past decade, numerous clinical data has been recorded on oral Candidiasis associated with oral piercings. Studies show that the prevalence of developing yeast deposits are more common on the surface of the pierced tongue, than on other oral surfaces.

According to the majority of described cases, the species identified were C. albicans, which is susceptible to treatment with common anti- fungal agents. With the increasing incidence of NCAC species, which are prone to the development of antifungal resistance, there is a persistent requirement in clinical research for developing newer and more effective agents, also differentiating the present yeast colonies, to determinate the best individual treatment options. Bacterial infections can be commonly associated to piercing insertion in the oral mucosa, especially the tongue. They can commonly be observed alongside oral thrush. Preventive measures should be taken for avoiding the procedure- related consequences.
Complications of Tongue Piercing: A Review of the Literature and Three Case Reports. The journal of contemporary dental practice; 2009.

3. Hardee P, Mallya L, Hutchison I. Case report: Tongue piercing resulting in hypotensive collapse. British Dental Journal. 2000;188:657-658.

4. Maibaum W, Margherita V. Tongue piercing: a concern for the dentist. General dentistry. 1999;45:495-7.

5. Francu L, Calin D. Lingual Piercing: Dental Anatomical Changes Induced By Trauma and Abrasion. Romanian Journal of Functional and Clinical, Macro- and Microscopical Anatomy and of Anthropology. 2012;11:7477.

6. Farah C, Harmon D. Tongue piercing: Case report and review of current practice. Australian dental journal. 1999;43:387-9

7. Ziebolz D, Hildebrand A, Proff P, Rinke S, Hornecker E, Mausberg R F. Long-term effects of tongue piercing--a case control study. Clinical oral investigations. 2012;16(1):231-237.

8. Bagnall S. Oral piercing \& dental complications. Vital 9, 2011;20-22.

9. Singh A, et al. Oral candidiasis: An overview. Journal of oral and maxillofacial pathology: JOMFP. 2014;18,Suppl $1 .$.

10. Akpan A, Morgan R. Oral candidiasis. Postgraduate medical journal. 2002;78:922.

11. Patil S, Rao Roopa S, Majumdar B, Anil S. Clinical Appearance of Oral Candida Infection and Therapeutic Strategies, Frontiers in Microbiology. 2011;6:1391.

12. Knoke M, Bernhardt H., The first description of an oesophageal candidosis by Bernhard von Langenbeck in 1839, Mycoses. $2006 \mathrm{Jul} ; 49(4): 283-7$.

13. Barnett JA. A history of research on yeasts 12: medical yeasts part 1, Candida albicans. Yeast. 2008;25:385-417.

14. Lalla RV, Patton LL, Dongari-Bagtzoglou A. Oral candidiasis: pathogenesis, clinical presentation, diagnosis and treatment strategies. J Calif Dent Assoc. 2013;41:263268.

15. Zadik Y, Burnstein S, Derazne E, Sandler V, Ianculovici C, Halperin T. Colonization of Candida: prevalence among tongue-pierced and non-pierced immunocompetent adults., Oral Dis. 2010 Mar;16(2):172-5.

16. $\mathrm{Yu} \mathrm{CH}$, Minnema BJ, Gold WL. Bacterial infections complicating tongue piercing. Can J Infect Dis Med Microbiol. 2010;21(1).

17. Inchingolo $\mathrm{F}$, Tatullo $\mathrm{M}$, Abenavoli FM, et al. Oral piercing and oral diseases: a short time retrospective study. International Journal of Medical Sciences. 2011;8(8):649652.

18. Rossie K, Guggenheimer J. Oral candidiasis: clinical manifestations, diagnosis, and treatment. Pract. Periodontics Aesthet Dent. 1997;9 635-641; quiz 642.

19. Gutierrez J, Morales P, Gonzalez M A, Quindos G. Candida dubliniensis, a new fungal pathogen. J. Basic Microbiol. 2002;42 207-227.

20. Thompson GR, Patel PK, Kirkpatrick WR, Westbrook SD, Berg D, Erlandsen J, et al. Oropharyngeal candidiasis in the era of antiretroviral therapy. Oral Surg. Oral Med. Oral Pathol Oral Radiol. Endod; 2010.

\section{REFERENCES}

1. Purim KSM, et al. Piercings in medical students and their effects on the skin. Anais brasileiros de dermatologia. 2014;89(6).

2. Ziebolz D, Von See C, Nüss K, Hornecke E, Mausberg R. 